\title{
Colesterol total y discapacidad en ancianos hospitalizados: más allá de la enfermedad cardiovascular
}

\author{
José Mauricio Ocampo ${ }^{\mathrm{a}, \mathrm{b}, *}$, Carlos A. Reyes-Ortiz ${ }^{\mathrm{c}}$, \\ Rafael Eduardo Escandón González ${ }^{a}$, María Eugenia Casanova ${ }^{d}$, \\ Marisol Badiel Ocampo ${ }^{\mathrm{e}, \mathrm{f}}$ y Juan Karlo Urrea ${ }^{\mathrm{a}}$
}

a Departamento de Medicina Interna, Universidad Libre, Cali, Colombia

b Departamento de Medicina Familiar, Universidad del Valle, Cali, Colombia

c Division of Geriatric and Palliative Medicine, Department of Internal Medicine, McGovern Medical School, University of Texas Health Science Center at Houston, USA

d Programa de Especialización en Medicina Interna, Universidad Libre, Cali, Colombia

e Ciencias Comunitarias, Universidad Libre, Cali, Colombia

${ }^{f}$ Departamento de Cirugía, Área de Investigación Clínica, Hospital Universitario del Valle, Subgerencia de Investigaciones, Cali, Colombia

Recibido el 11 de mayo de 2017; aceptado el 5 de agosto de 2017

Disponible en Internet el 1 de diciembre de 2017

\section{PALABRAS CLAVE}

Anciano;

Colesterol;

Discapacidad;

Hospitalización

\begin{abstract}
Resumen
Objetivo: determinar si los niveles de colesterol total predicen recuperación en actividades básicas de la vida diaria.

Métodos: estudio de cohorte prospectiva que incluyó 806 pacientes $\geq 60$ años, hospitalizados en la unidad geriátrica de agudos entre julio de 2013 y agosto de 2015. El desenlace fue desarrollo de discapacidad evaluada mediante el índice de Barthel $\leq 60$ en cuatro momentos: 15 días antes del ingreso, al ingreso, al egreso y 30 días después. El colesterol total fue dividido en cuartiles. Se incluyeron variables demográficas, sociales y clínicas. Se usaron los modelos de regresión logística multivariada para predecir discapacidad y mortalidad, junto con el procedimiento Glimmix para análisis longitudinales y para la predicción del índice de Barthel total.
\end{abstract}

\footnotetext{
* Autor para correspondencia.

Correo electrónico: jmocampo2000@yahoo.com.ar (J.M. Ocampo).
} 
Resultados: la edad fue $82,3 \pm 7,2$. El 52\% eran mujeres. En el análisis multivariado la discapacidad estuvo asociada con edad $\geq 85$ años, alta comorbilidad, deterioro social, hipoalbuminemia, MMSE < 19 y delirium. Los cuartiles más altos de colesterol total (Q2, Q3, Q4) tuvieron menor riesgo para discapacidad que el cuartil más bajo (Q1). El análisis longitudinal mostró disminución del índice de Barthel en pacientes con estancia hospitalaria > 15 días, hipoalbuminemia, MMSE < 19 y delirium. Los cuartiles más altos de colesterol total alcanzan mayor índice de Barthel a través del tiempo en comparación con el Q1. La mortalidad estuvo asociada con alta comorbilidad, índice de Barthel $\leq 60$ al ingreso y $M M S E<19$. Los niveles de colesterol total no se asociaron con mortalidad.

Conclusiones: el colesterol total en los cuartiles más altos estuvo asociado con mejoría en la recuperación de las actividades básicas de la vida diaria desde 15 días antes del ingreso hospitalario hasta 30 días después de alta, comparado con el Q1.

(C) 2017 Sociedad Colombiana de Cardiología y Cirugía Cardiovascular. Publicado por Elsevier España, S.L.U. Este es un artículo Open Access bajo la licencia CC BY-NC-ND (http:// creativecommons.org/licenses/by-nc-nd/4.0/).

\section{KEYWORDS \\ Elderly; \\ Cholesterol; \\ Disability; \\ Hospital admissions}

\section{Total cholesterol and disability in the hospitalised elderly: beyond cardiovascular disease}

\begin{abstract}
Objective: To determine whether the total cholesterol levels predict recovery in basic activities of daily living.

Methods: A prospective cohort study was conducted on 806 patients $\geq 60$ years-old, admitted into an Acute Geriatric Unit between July 2013 and August 2015. The outcome was development of a disability assessed by a Barthel index $\leq 60$ at four different times: 15 days before admission, on admission, at discharge, and 30 days afterwards. The total cholesterol was divided into quartiles. A record was made of the social and clinical demographic variables. Multivariate logistic regression models were used to predict disability and mortality, together with the Glimmix procedure for the longitudinal analysis and for the prediction of the total Barthel index.

Results: The mean age of the patients was $82.3 \pm 7.2$ years, and $52 \%$ were women. In the multivariate analysis, disability was associated with an age $\geq 85$ years, high comorbidity, social impairment, low plasma albumin, $M M S E<19$, and delirium. The highest total cholesterol quartiles (Q2, Q3, Q4) had a lower risk for disability than the lowest quartile (Q1). The longitudinal analysis showed a decrease in the Barthel index in patients with a hospital stay of $>15$ days, low plasma albumin, MMSE $<19$, and delirium. The highest total cholesterol quartiles reach a higher Barthel index over time compared with the lowest quartile. The mortality was associated with a high comorbidity, a Barthel index $\leq 60$ on admission, and MMSE $<19$. The total cholesterol levels were not associated with mortality.

Conclusions: The total cholesterol in the highest quartiles was associated with an improvement in the recovery of basic activities of daily living from 15 days before hospital admission up to 30 days after discharge compared with the lowest quartile (Q1).

(c) 2017 Sociedad Colombiana de Cardiología y Cirugía Cardiovascular. Published by Elsevier España, S.L.U. This is an open access article under the CC BY-NC-ND license (http:// creativecommons.org/licenses/by-nc-nd/4.0/).
\end{abstract}

\section{Introducción}

El colesterol total es un lípido sintetizado en diferentes tejidos corporales, en particular en el hígado y la pared intestinal. Aproximadamente, un $75 \%$ se produce en el organismo y el restante se obtiene de los alimentos ${ }^{1}$.

Pese a que la evidencia consolidada muestra cómo los niveles de colesterol total en individuos jóvenes y adultos son un predictor independiente para el desarrollo de enfermedad y muerte cardiovascular, la importancia de sus valores elevados como factor de riesgo en ancianos todavía es controversial ${ }^{2}$.
Estudios transversales y de cohorte han demostrado un descenso progresivo de los niveles de colesterol total en mayores de 65 años $^{3}$, diferencias entre jóvenes y ancianos que posiblemente se expliquen por la prevalencia alta de enfermedades crónicas que afectan el estado de salud y condición funcional del último grupo ${ }^{4}$. Por ejemplo, los ancianos que presentan alta comorbilidad, discapacidad para realizar actividades básicas de la vida diaria y se encuentran institucionalizados, muestran niveles bajos de coleterol total ${ }^{3}$. Además, la presencia de enfermedades en estado subclínico asociadas con inflamación, también puede causar hipocolesterolemia debido al efecto de las 
citocinas proinflamatorias ${ }^{5}$. Con base en lo anterior, un estado de salud pobre puede comportarse como un factor distractor para la asociación entre niveles de colesterol total y desenlaces adversos en salud en ancianos, como discapacidad, institucionalización, mayor necesidad de cuidado formal e informal y muerte.

Asimismo, algunas investigaciones han evaluado las causas del descenso en los niveles de colesterol total en ancianos y han mostrado asociación con prevalencia alta de enfermedad crónica, modificaciones en el peso corporal, estilos de vida como consumo de alcohol y tabaco, empleo de medicamentos y efecto de supervivencia selectiva; este último se explica porque las personas con niveles altos de colesterol tienen mayor riesgo de muerte cardiovascular en edades tempranas, por tanto la probabilidad de encontrar ancianos con niveles altos de colesterol total es baja ${ }^{6}$.

Karlamangla et al. ${ }^{7}$, mostraron en una población de ancianos con alto nivel funcional, después de ajustar por potenciales distractores que incluían marcadores de condición de salud deficiente, que los niveles altos de colesterol total estaban asociados con buen estado de salud y baja probabilidad para desarrollar discapacidad en las actividades básicas de la vida diaria. Este hallazgo concuerda con un estudio de cohorte de Vauthey et al. ${ }^{8}$, en el que ancianos con niveles altos de colesterol total tuvieron menor probabilidad para desarrollar discapacidad después de una enfermedad cerebrovascular.

El proceso de discapacidad es el resultado de una alteración progresiva en la capacidad homeostática del organismo, $y$, es por tanto, una secuencia compleja de eventos en la que la fragilidad y la enfermedad crónica desempeñan un papel decisivo 9 .

En general, el proceso de discapacidad se ha considerado estático; no obstante, en los ancianos frágiles es una condición dinámica y heterogénea ${ }^{10}$. Estudios de cohortes muestran que una vez un porcentaje importante de ancianos desarrolla discapacidad, pueden recuperar las actividades básicas de la vida diaria, lo cual sugiere que se trata de un proceso complejo, con múltiples episodios de discapacidad interrelacionados que ocurren incluso en tiempos cortos como durante una hospitalización por enfermedad aguda ${ }^{11}$.

Los ancianos que requieren atención hospitalaria se caracterizan por ser un grupo heterogéneo y vulnerable debido a múltiples compromisos en las esferas biopsicosociales, como edad avanzada, disminución de la reserva funcional, múltiples enfermedades crónicas, polifarmacia, alteraciones cognoscitivas, compromiso funcional y baja red de apoyo social y familiar. Estas características se asocian con mayor riesgo para desarrollar discapacidad, como producto de la carga acumulada de envejecimiento, fragilidad y enfermedad ${ }^{12}$.

A diferencia de las unidades de atención hospitalaria convencional, las unidades geriátricas de agudos se basan en la prehabilitación y se enfocan en la condición funcional del anciano frágil, donde la atención es brindada por un equipo interdisciplinario. Las unidades geriátricas de agudos se han diseñado específicamente para prevenir la discapacidad y los eventos adversos relacionados con la hospitalización por enfermedad aguda o crónica reagudizada ${ }^{13}$.

Aunque pocos estudios han evaluado la asociación entre niveles de colesterol total y discapacidad, sobre todo en ancianos hospitalizados, no se conoce cuál es el comportamiento de la interacción entre estas dos variables cuando los pacientes son atendidos bajo el modelo de unidades geriátricas de agudos. El objetivo de la investigación fue determinar en una cohorte de ancianos frágiles hospitalizados en una unidad geriátrica de agudos, si los niveles de colesterol total predicen recuperación de las actividades básicas de la vida diaria independientemente de la comorbilidad y otros indicadores del estado de salud.

\section{Métodos}

\section{Diseño del estudio y recolección de la información}

Estudio de cohorte prospectiva en el que se admitieron participantes de forma consecutiva a la unidad geriátrica de agudos de la Clínica Universitaria Rafael Uribe Uribe en Cali, Colombia, entre julio de 2013 y agosto de 2015.

Se incluyeron pacientes de edad mayor a 60 años, consumo de más de ocho fármacos, enfermedad cerebrovascular en etapas subagudas, enfermedades incapacitantes y condición funcional previa de dependencia severa o total [puntaje en índice de Barthel $\leq 60$, readmisiones frecuentes (dos o más al mes), condiciones mentales previas (delirium al ingreso o demencia de base), presencia de múltiples síndromes geriátricos (fragilidad, úlceras por presión, caídas), índice de masa corporal de $<20 \mathrm{~kg} / \mathrm{m}^{2}$, redes de apoyo social insuficientes, procedencia de instituciones geriátricas $0>80$ años con enfermedad médica aguda].

Después de obtener el consentimiento informado por cada paciente, un médico con entrenamiento completó el cuestionario al momento del ingreso hospitalario. Se realizó una sesión de entrenamiento a los profesionales del equipo interdisciplinario con el fin de recolectar la información estandarizada de escalas de valoración geriátrica integral.

La recolección de la información incluyó variables demográficas, sociales, valoración geriátrica integral e información clínica. Los pacientes o algún informante proxi se entrevistaron para obtener la información al ingreso y alta hospitalaria y seguimiento telefónico al mes después del alta.

De las 905 personas atendidas en la unidad geriátrica de agudos, se excluyeron 40 por datos perdidos en colesterol y 59 por información de funcionalidad; la población final del estudio fue de 806 pacientes.

El protocolo del estudio fue aprobado por el Comité de Ética y Científico de la Investigación Subdirección Educación Superior e Investigación de la Corporación Comfenalco ValleUniversidad Libre, Clínica Universitaria Rafael Uribe Uribe.

\section{Desenlace}

Discapacidad durante el proceso de la enfermedad aguda evaluado mediante el Índice de Barthel en cuatro momentos: 15 días antes del ingreso hospitalario, al ingreso y egreso hospitalario y 30 días después del alta hospitalaria. 


\section{Variables de estudio}

El colesterol total sérico se determinó a través del método enzimático colorimétrico basado en la reacción de Trinder realizado en el laboratorio de la institución. Para la valoración del estado funcional se utilizó el índice de Barthel ${ }^{14}$ que evalúa 10 actividades básicas de la vida diaria, asignándole un valor predeterminado para la autonomía/independencia. La puntuación varía de 0 a 100; 0 es la máxima dependencia y 100 la independencia total.

Durante el tiempo de estancia hospitalaria en la unidad geriátrica de agudos se hizo valoración geriátrica integral a cargo del equipo interdisciplinario coordinado por un médico geriatra. El estado cognitivo se evaluó con la escala del examen mínimo mental [Mini-Mental State Examination (MMSE) $]^{15}$. Los participantes se agruparon según el puntaje como: normal $\geq 24$, leve 19 a 23 , moderado 14 a 18 y severo $<14$.

La presencia de delirium se valoró con la escala CAM (Confusion Assessment Method), diseñada por Inouye et al. ${ }^{16}$ para el diagnóstico específico de delirium con base en los criterios diagnósticos del DSM-III-R.

La comorbilidad se midió con el índice de comorbilidad de Charlson. Se consideró ausencia de comorbilidad entre 0 y 1 punto, comorbilidad baja cuando el índice es 2 y alta comorbilidad cuando es igual o superior a 3 puntos $^{17}$.

Para evaluar la condición sociofamiliar se aplicó la escala de valoración de Gijón abreviada y modificada. Esta evalúa situación familiar, relaciones y contactos sociales y apoyos de red social ${ }^{18}$. La condición sociofamiliar se categorizó según la puntuación como buena $\leq 7$, en riesgo 8-9 y de deterioro social severo $\geq 10$.

\section{Covariables}

En los análisis se incluyeron edad, sexo y variables conocidas que pudieran afectar el desenlace. La anemia se definió según los criterios de la Organización Mundial de la Salud: para mujeres hemoglobina $<12 \mathrm{~g} / \mathrm{dl}$ y para hombres $<13 \mathrm{~g} / \mathrm{d}^{19}$. La creatinina plasmática se determinó a través del método de medida enzimático (creatininasa) hecho en el laboratorio de institución, el cual presenta una especificidad analítica superior al de Jaffé, por ser menos sensible a las interferencias por pseudocromógenos ${ }^{20}$. La medición de la creatinina se realizó una vez el paciente se encontraba en condiciones clínicas estables. Se consideró como un índice de pobre estado nutricional una hipoalbuminemia definida como albúmina sérica $<3,5 \mathrm{~g} / \mathrm{dl}$.

\section{Análisis estadístico}

Todos los análisis se hicieron en el programa estadístico SAS versión 9.4 para Windows (SAS Institute, Inc., Cary, NC); el nivel de significancia estadística seleccionado fue $p<0,05$, para la prueba de dos colas. Se realizó un análisis exploratorio y descriptivo. Las variables continuas se expresan en promedios y desviación estándar o medianas y rangos intercuartiles según la distribución de los datos. Se estimaron proporciones para las variables categóricas.

Para el análisis bivariado entre las variables independientes con la variable colesterol según los valores de cuartiles, se empleó la prueba de $\mathrm{Chi}^{2}$ o prueba de Fisher, según el caso, para las variables categóricas, y mediante análisis de varianza (ANOVA) para las variables cuantitativas.

Se realizó un modelo multivariado de regresión logística para determinar la asociación entre el estado funcional evaluado mediante el índice de Barthel $\leq 60$, valor que ha mostrado asociación con dependencia para realizar actividades básicas de la vida diaria y puede predecir deterioro funcional, mortalidad, estancia hospitalaria, ayuda social e institucionalización ${ }^{21}$. Se usaron como categorías de referencia: edad $\geq 85$ años, sexo femenino, índice de Charlson > 3, MMSE agrupado en moderado y severo ( $<19$ puntos), delirium, situación de deterioro social severo, anemia e hipoalbuminemia, para obtener un estimado de la razón de probabilidad (OR) con su respectivo intervalo de confianza del 95\% (IC95\%). De manera similar, se usó la regresión logística para determinar variables asociadas con la mortalidad, donde se ajustaron las más relevantes dentro del estudio y de acuerdo con la literatura.

Con el propósito de describir la relación entre la variable colesterol total según cuartiles con los puntajes de índice de Barthel total en el tiempo, se realizó una gráfica en Excel $^{\oplus}$, en la que se usaron valores basales de 15 días antes (tiempo 1) y de seguimiento [tiempos 2 (ingreso), 3 (alta) y 4 (seguimiento)].

En los análisis longitudinales se tuvieron en cuenta los datos no balanceados (participantes con al menos un seguimiento en la medición del índice de Barthel total: basal $=15$ días antes y al menos uno de los seguimientos, contribuyeron para los cálculos longitudinales) y los modelos para los cambios en las trayectorias funcionales en el tiempo (mediciones de actividades repetidas). Para probar si en el tiempo las variables independientes estaban asociadas con el índice de Barthel total (medidas repetidas durante los tiempos 1, 2, 3 y 4), y como el índice de Barthel no presentó una distribución normal, se usó el procedimiento Glimmix en SAS. Antes del análisis se hizo una transformación estadística del valor del Barthel para obtener una distribución beta y así usar el procedimiento Glimmix, en forma análoga como lo realizaron Hunger et al. ${ }^{22}$ Así, se obtuvieron estimadores (de interpretación equivalente a los coeficientes beta en modelos de regresión lineal) con errores estándar, donde la variable tiempo muestra disminución de la variable dependiente (índice de Barthel total) durante el período de seguimiento y un signo positivo en el estimador indica una asociación directa de la variable independiente con el índice de Barthel a través del tiempo; en contraste, un signo negativo indica una asociación inversa del índice de Barthel a través del tiempo (p.e., la presencia de alta comorbilidad puede determinar un menor puntaje de índice de Barthel al final).

\section{Resultados}

806 pacientes cumplieron los criterios de selección. La edad promedio fue $82,3 \pm 7,2$ años, siendo el grupo de menores de 85 años el más frecuente (66\%); $52 \%$ son del género femenino; $91 \%$ presentó una estancia no mayor a 30 días y de estos el promedio fue $11 \pm 6,7$ días; $5 \%$ falleció al egreso (tabla 1 ).

El promedio de colesterol total en la población fue $136,2 \pm 37,9 \mathrm{mg} / \mathrm{dl}$; para los $\leq 84$ años fue 
Tabla 1 Características sociodemográficas de la población de estudio

\begin{tabular}{ll}
\hline Variable & Total (806) \\
\hline $\begin{array}{l}\text { Edad, años } \\
\quad \text { Promedio } \pm \text { DE }\end{array}$ & $82,3 \pm 7,2$ \\
$\begin{array}{l}\text { Grupos de edad, } n(\%) \\
\quad \leq 84\end{array}$ & $533(66)$ \\
$\quad 85-105$ & $273(34)$ \\
$\quad$ Género (\%) & \\
$\quad$ Femenino & $419(52)$ \\
Estancia hospitalaria, días & \\
$\quad$ Promedio \pm DE & $11,1 \pm 6,7$ \\
Estado al egreso (\%) & \\
$\quad$ Muerto & $40(5)$ \\
\hline
\end{tabular}

$137,5 \pm 39,4 \mathrm{mg} / \mathrm{dl}$, y $133,1 \pm 34,2 \mathrm{mg} / \mathrm{dl}$ para los de $\geq 85$ años; $134,5 \pm 39,5 \mathrm{mg} / \mathrm{dl}$ para los hombres $y$ $137,7 \pm 36,4 \mathrm{mg} / \mathrm{dl}$ para las mujeres. Los cuartiles de colesterol total se distribuyeron de la siguiente forma: Q1 90,9 $\pm 13,2$ (rango 50-107); Q2 117,3 \pm 5,9 (rango 108-129); Q3 142,2 $\pm 7,9$ (rango 130-156); Q4 183,9 $\pm 26,2$ (rango 157-325).

La estancia promedio de los pacientes con niveles más bajos de colesterol total fue estadísticamente diferente a la de los pacientes con niveles más altos de colesterol total. Los pacientes con valores más bajos de colesterol total presentaron mayor declinación funcional, dependencia, comorbilidades, demencia, inflamación y niveles más bajos de albúmina en comparación con los de los niveles más elevados.

El análisis bivariado mostró que la estancia promedio de los pacientes del Q1 fue estadísticamente diferente a la de los pacientes del Q4 (12 vs. 10,2). Se tomó la estancia con punto de corte a 30 días porque menos del $10 \%$ tuvo estancias prolongadas (tabla 2).
La diferencia promedio del índice de Barthel inicial en pacientes con colesterol total bajo cuyo promedio fue $90 \mathrm{mg} / \mathrm{dl}(\mathrm{Q} 1)$, es estadísticamente diferente respecto a los pacientes de los demás cuartiles que tuvieron valores de colesterol total superiores en promedio $(15,4,19,21$ respectivamente) (tabla 3). El puntaje promedio de Lawton en los pacientes del Q1 fue estadísticamente diferente al puntaje promedio de los pacientes del Q3 y Q4. Los pacientes del Q1 presentaron bajos niveles de albúmina estadísticamente diferentes a los de los demás pacientes del Q2, Q3 y Q4.

De igual forma, los ancianos con bajos niveles de colesterol total, se asociaron con menores niveles de albúmina $(p<0,000)$, mayor comorbilidad $(p<0,0009)$, menores niveles de hemoglobina $(p<0,000)$, menor conteo $(<1.500)$ de linfocitos $(p=0,04)$ y mayor inflamación $P C R$ $(p=0,001)$, además de minimental severo $(p=0,049)$, delirium $(p=0,021)$ y mayor depresión $(p=0,007)$.

La tabla 4 enumera las variables asociadas con índice de Barthel $\leq 60$ luego de ajustes en el análisis multivariado; en los $\geq 85$ años $(O R=1 ; 35)$ se encontró alta comorbilidad $(O R=1,4)$, deterioro social $(O R=1,47)$, hipoalbuminemia $(O R=2,6), M M S E<19(O R=3,0)$ y delirium $(O R=3,8)$ que aumentan el riesgo de discapacidad al ingreso hospitalario, mientras que los cuartiles más altos de colesterol total (Q2, Q3, Q4) tienen menor riesgo que el cuartil más bajo (Q1) para desarrollar discapacidad $(\mathrm{OR}<1)$.

La tabla 5 muestra las variables asociadas con el índice de Barthel total luego de ajustes del análisis multivariado longitudinal (tiempos 1 a 4); se observa una disminución del índice de Barthel a través del tiempo. así como del índice de Barthel total (betas negativas) en pacientes con estancia hospitalaria $>15$ días, MMSE $<19$, delirium e hipoalbuminemia, mientras que los cuartiles más altos de colesterol total (Q2, Q3, Q4) alcanzan mayor índice de Barthel a través del tiempo (betas positivas), en comparación con los del cuartil más bajo (Q1).

La figura 1 muestra que los pacientes con función del cuartil más bajo de colesterol total (Q1) tienen niveles de índice de Barthel más bajos que las otras categorías.

Tabla 2 Características sociodemográficas según niveles de colesterol total por cuartiles

\begin{tabular}{|c|c|c|c|c|c|}
\hline Variable & $\begin{array}{l}\text { Q1 (n= 202) } \\
{ }^{*} 90 \mathrm{mg} / \mathrm{dl}\end{array}$ & $\begin{array}{l}\mathrm{Q} 2(\mathrm{n}=201) \\
{ }^{*} 117 \mathrm{mg} / \mathrm{dl}\end{array}$ & $\begin{array}{l}\text { Q3 (n= 202) } \\
{ }^{*} 142 \mathrm{mg} / \mathrm{dl}\end{array}$ & $\begin{array}{l}\text { Q4 (n= 201) } \\
{ }^{*} 185 \mathrm{mg} / \mathrm{dl}\end{array}$ & Valor $\mathrm{p}$ \\
\hline Edad, promedio $\pm D E$ & $81 \pm 7$ & $82 \pm 6,9$ & $82 \pm 6,7$ & $81 \pm 7$ & $0,071^{a}$ \\
\hline \multicolumn{6}{|l|}{ Grupos de edad, (\%) } \\
\hline$\leq 84$ & 68,81 & 65,67 & 60,40 & 69,65 & $0,19^{b}$ \\
\hline $85-105$ & 31,19 & 34,33 & 39,60 & 30,35 & \\
\hline \multicolumn{6}{|l|}{ Género (\%) } \\
\hline Femenino & 49,50 & 48,76 & 54,95 & 54,73 & $0,448^{b}$ \\
\hline \multicolumn{6}{|c|}{ Estancia hospitalaria, días } \\
\hline Promedio $\pm \mathrm{DE}$ & $12 \pm 7,5$ & $11,5 \pm 6,9$ & $10,6 \pm 6,3$ & $10,2 \pm 6,1$ & $0,025^{a}$ \\
\hline \multicolumn{6}{|l|}{ Estado al egreso (\%) } \\
\hline Muerto & 7,04 & 3,78 & 5,76 & 4,12 & $0,442^{\mathrm{b}}$ \\
\hline
\end{tabular}


Tabla 3 Características funcionales, biológicas y mentales según niveles de colesterol total por cuartiles

\begin{tabular}{|c|c|c|c|c|c|}
\hline Característica & $\begin{array}{l}\text { Q1 }(n=202) \\
{ }^{*} 90 \mathrm{mg} / \mathrm{dl}\end{array}$ & $\begin{array}{l}\mathrm{Q} 2(\mathrm{n}=201) \\
{ }^{*} 117 \mathrm{mg} / \mathrm{dl}\end{array}$ & $\begin{array}{l}\text { Q3 }(\mathrm{n}=202) \\
\text { "142 mg/dl }\end{array}$ & $\begin{array}{l}\mathrm{Q} 4(\mathrm{n}=201) \\
{ }^{*} 185 \mathrm{mg} / \mathrm{dl}\end{array}$ & Valor $\mathrm{p}$ \\
\hline \multicolumn{6}{|l|}{ Funcionales Promedio $\pm D E$} \\
\hline Barthel al ingreso & $37 \pm 38$ & $52 \pm 39$ & $56 \pm 39$ & $58 \pm 39$ & $<0,000^{a}$ \\
\hline Lawton al ingreso & $2 \pm 2,6$ & $2,5 \pm 2,6$ & $2,9 \pm 3,1$ & $3,1 \pm 3$ & $0,0014^{a}$ \\
\hline \multicolumn{6}{|l|}{ Biológicas Promedio $\pm D E$} \\
\hline Glicemia & $161 \pm 101,4$ & $137 \pm 68,2$ & $137 \pm 70$ & $152 \pm 85,5$ & $0,045^{a}$ \\
\hline Albúmina & $3,1 \pm 0,8$ & $3,2 \pm 0,5$ & $3,35 \pm 0,5$ & $3,4 \pm 0,5$ & $<0,000^{a}$ \\
\hline Índice de Charlson & $3,9 \pm 2,1$ & $3,3 \pm 2$ & $3,4 \pm 2,1$ & $3,1 \pm 1,8$ & $0,0009^{a}$ \\
\hline \multicolumn{6}{|l|}{ Charlson categorizado (\%) } \\
\hline Sin comorbilidad & 11,88 & 16,92 & 17,82 & 16,42 & $0,11^{\mathrm{b}}$ \\
\hline Baja comorbilidad & 15,35 & 23,88 & 19,31 & 22,39 & \\
\hline Alta comorbilidad & 72,77 & 59,20 & 62,87 & 61,19 & \\
\hline Hemoglobina & $11,1 \pm 2,5$ & $11,9 \pm 2,2$ & $11,9 \pm 2,1$ & $12,1 \pm 2$ & $<0,000^{a}$ \\
\hline Creatinina & $1,5 \pm 1,6$ & $1,3 \pm 0,9$ & $1,2 \pm 0,8$ & $1,4 \pm 1,3$ & $0,11^{\mathrm{a}}$ \\
\hline Linfocitos $\leq 1.500(\%)$ & 45,21 & 50,26 & 51,30 & 59,69 & $0,04^{b}$ \\
\hline Sodio & $138,1 \pm 6,7$ & $138 \pm 8$ & $138,6 \pm 6,2$ & $137,7 \pm 7,2$ & $0,63^{a}$ \\
\hline PCR & $79,3 \pm 86$ & $68,7 \pm 80,4$ & $60,9 \pm 80,6$ & $45,6 \pm 70,9$ & $0,001^{a}$ \\
\hline \multicolumn{6}{|l|}{ Mentales } \\
\hline \multicolumn{6}{|l|}{ Minimental (\%) } \\
\hline Normal & 17,8 & 20,4 & 26,2 & 28,3 & $0,049^{b}$ \\
\hline Leve & 8,6 & 10,9 & 12 & 16 & \\
\hline Moderado & 29,6 & 37,4 & 29 & 27 & \\
\hline Severo & 44,2 & 31,3 & 33 & 29 & \\
\hline Delirium (\%) & 35,3 & 21,8 & 22,7 & 23,6 & $0,021^{\mathrm{b}}$ \\
\hline \multicolumn{6}{|l|}{ Social } \\
\hline \multicolumn{6}{|l|}{ Guijón } \\
\hline Buena & 47,85 & 60,00 & 59,04 & 57,56 & \\
\hline Deterioro social & 11,66 & 8,13 & 10,24 & 8,72 & $0,34^{b}$ \\
\hline Intermedia & 40,49 & 31,87 & 30,72 & 33,72 & \\
\hline
\end{tabular}

Tabla 4 Niveles de colesterol total por cuartiles asociados con índice de Barthel $\leq 60$ al ingreso hospitalario, regresión logística multivariada $(n=806)$

\begin{tabular}{|c|c|c|c|c|}
\hline Variables & OR & & IC95\% & Valor $\mathrm{p}$ \\
\hline Edad $\geq 85$ años & 1,350 & 1,045 & 1,745 & 0,0215 \\
\hline Mujer & 1,255 & 0,980 & 1,607 & 0,0720 \\
\hline Alta comorbilidad & 1,430 & 1,116 & 1,834 & 0,0047 \\
\hline Deterioro social severo & 1,470 & 1,127 & 1,917 & 0,0044 \\
\hline Hipoalbuminemia & 2,656 & 1,859 & 3,794 & $<, 0001$ \\
\hline Anemia & 1,293 & 0,990 & 1,689 & 0,0592 \\
\hline MMSE $<19$ & 3,034 & 2,319 & 3,970 & $<, 0001$ \\
\hline Delirium & 3,827 & 2,755 & 5,317 & $<, 0001$ \\
\hline \multicolumn{5}{|l|}{ Cuartiles de colesterol } \\
\hline Colesterol Q1 (referencia) & 1,000 & & & \\
\hline Colesterol Q2 & 0,604 & 0,401 & 0,909 & 0,0158 \\
\hline Colesterol Q3 & 0,499 & 0,326 & 0,762 & 0,0013 \\
\hline Colesterol Q4 & 0,616 & 0,405 & 0,937 & 0,0236 \\
\hline
\end{tabular}


Tabla 5 Niveles de colesterol total por cuartiles asociados con índice de Barthel total durante los tiempos 1, 2, 3, 4 regresión longitudinal (Proc Glimmix en SAS) multivariada $(n=806)$

\begin{tabular}{llll}
\hline Efecto & Estimado (beta) & Error estándar & Valor $p$ \\
\hline Intercepto & $-0,4514$ & 0,3108 & 0,1470 \\
Tiempo & $-0,07618$ & 0,02598 & 0,0034 \\
Edad $\geq 85$ años & $-0,1426$ & 0,1355 & 0,2932 \\
Mujer & $-0,1576$ & 0,1246 & 0,2066 \\
Deterioro social severo & $-0,1363$ & 0,1384 & 0,3252 \\
Estancia hospitalaria $\geq 15$ días & $-0,8314$ & 0,1378 & $<, 0001$ \\
Alta comorbilidad & $-0,1417$ & 0,1316 & 0,2821 \\
MMSE $<19$ & $-0,8182$ & 0,1546 & $<, 0001$ \\
Delirium & $-1,1035$ & 0,1551 & $<, 0001$ \\
Hipoalbuminemia & $-0,7806$ & 0,1520 & $<, 0001$ \\
Cuartiles de cholesterol & & & \\
$\quad$ Colesterol Q1 (referencia) & 0 & & 0,0012 \\
Colesterol Q2 & 0,5821 & 0,1793 & 0,0328 \\
Colesterol Q3 & 0,7737 & 0,1778 & 0,1782 \\
Colesterol Q4 & 0,3813 & & \\
\hline
\end{tabular}

Una beta negativa indica disminución del índice de Barthel Total; una beta positiva indica aumento del índice de Barthel total.



Figura 1 Modificación en los puntajes promedio e IC95\% del índice de Barthel para cada uno de los cuatro tiempos de valoración según niveles de colesterol total por cuartiles.

Llama la atención que los mejores índices de Barthel al seguimiento están en los sujetos con los cuartiles intermedios (Q2, Q3).

La tabla 6 presenta las variables asociadas con mortalidad haciendo ajustes en el análisis multivariado; el género femenino se asocia con menor mortalidad en forma significativa, en tanto que hay mayor mortalidad asociada con alta comorbilidad, índice de Barthel $\leq 60$ al ingreso hospitalario y MMSE < 19. Los niveles de colesterol total no se asociaron con la mortalidad.

\section{Discusión}

Este es el primer estudio que evalúa la relación entre niveles de colesterol total y recuperación de las actividades básicas de la vida diaria en un tiempo corto, en una cohorte de ancianos frágiles hospitalizados en unidades geriátricas de agudos atendidos por un equipo interdisciplinario.
El estudio muestra que ancianos frágiles hospitalizados en una unidad geriátrica de agudos y niveles elevados de colesterol total predicen de forma independiente una mayor probabilidad de recuperación de la discapacidad en actividades básicas de la vida diaria.

Se ha documentado que los pacientes hospitalizados tienen menor nivel de colesterol total que personas saludables ${ }^{6}$, hallazgos similares encontrados en el estudio, donde el promedio en los niveles de colesterol total de los pacientes fue bajo, probablemente como resultado de la alta comorbilidad y otras variables como albúmina, hemoglobina y proteína $\mathrm{C}$ reactiva (tabla 2 ).

En el análisis de regresión logística multivariada (tabla 4) se documentó que el estado funcional de los ancianos hospitalizados evaluados mediante el índice de Barthel al ingreso hospitalario, es una variable que no solo depende de factores biológicos como edad y comorbilidad, sino también de variables clínicas como función renal, niveles de colesterol total y estado nutricional (hipoalbuminemia), factores 
Tabla 6 Factores asociados con mortalidad, regresión logística multivariada $(n=806)$

\begin{tabular}{|c|c|c|c|c|}
\hline \multirow{2}{*}{$\begin{array}{l}\text { Variables } \\
\text { Edad } \geq 85 \text { años }\end{array}$} & \multirow{2}{*}{$\frac{\mathrm{OR}}{0,978}$} & \multicolumn{2}{|c|}{ IC95\% Val } & \multirow{2}{*}{$\frac{\text { or } p}{06}$} \\
\hline & & 0,662 & $1,4450,91$ & \\
\hline Mujer & 0,595 & 0,403 & $0,8790,00$ & 91 \\
\hline Alta comorbilidad & 1,774 & 1,157 & $2,7220,00$ & 86 \\
\hline Barthel $\leq 60$ & 4,494 & 2,306 & $8,757<, 000$ & 1 \\
\hline Estancia $\geq 15$ días & 1,031 & 0,698 & $1,5220,87$ & 99 \\
\hline Deterioro social severo & 1,257 & 0,806 & $1,9610,31$ & 28 \\
\hline Hipoalbuminemia & 0,600 & 0,316 & $1,1410,11$ & 92 \\
\hline Anemia & 0,860 & 0,552 & $1,3410,50$ & 68 \\
\hline MMSE $<19$ & 0,369 & 0,163 & $0,8340,01$ & 66 \\
\hline \multicolumn{5}{|l|}{ Cuartiles de colesterol } \\
\hline Colesterol Q1 (referencia) & 1,000 & & & \\
\hline Colesterol Q2 & 0,995 & 0,488 & $2,0290,98$ & 86 \\
\hline Colesterol Q3 & 0,499 & 0,207 & $1,2030,12$ & 16 \\
\hline Colesterol Q4 & 0,915 & 0,492 & $1,7020.77$ & 99 \\
\hline
\end{tabular}

mentales como demencia y delirium, además de factores sociofamiliares. Lo anterior resalta la importancia de hospitalizar al anciano en una unidad geriátrica de agudos en la que se le realice una valoración geriátrica integral que se fundamente en el modelo biopsicosocial y se haga mediante la metodología de trabajo del equipo interdisciplinar, a diferencia del modelo tradicional de atención donde sólo se hace énfasis en la enfermedad ${ }^{13}$. Ellis et al. ${ }^{23}$, demostraron que el manejo de los ancianos en una unidad geriátrica de agudos a diferencia de las unidades convencionales de atención, se asocia con mayor probabilidad de vivir en casa al alta y a los 6 meses siguientes, disminución en la mortalidad, institucionalización y discapacidad para las actividades básicas de la vida diaria al alta y mejoría en la cognición. Diferentes estudios epidemiológicos y clínicos han demostrado asociación entre niveles bajos de colesterol total y pobre estado de salud en ancianos ${ }^{6}$. Se ha encontrado que en ancianos frágiles es frecuente encontrar niveles bajos de colesterol total que los hace buenos predictores para mortalidad, aunque esta asociación podría ser dependiente del efecto de la comorbilidad para disminuir los niveles de colesterol total ${ }^{24}$. Los resultados mostraron que a mayor nivel de colesterol total existía menor probabilidad para presentar discapacidad en las actividades básicas de la vida diaria al ingreso hospitalario independientemente del sexo, la edad, la comorbilidad, la demencia, el delirium y la condición sociofamiliar (tabla 4); lo anterior confirma los hallazgos documentados en estudios previos a nivel hospitalario ${ }^{25}$.

De otra parte, el hallazgo de mayores niveles de colesterol total con mejor valor en el índice de Barthel durante los cuatro tiempos de observación, concuerda con estudios de cohorte previos realizados en ancianos, en los que se encontró que niveles elevados de colesterol total se asociaron con bajo riesgo para desarrollar discapacidad en actividades básicas de la vida diaria y mortalidad posterior a enfermedad cerebrovascular ${ }^{8}$.

En el análisis de regresión longitudinal, los cuartiles de colesterol total (Q2 a Q4) predijeron un mejor índice de Barthel para los cuatro tiempos de observación, lo cual sugiere la existencia de un nivel de umbral y que un incremento mayor a la categoría superior no mejora o disminuye el valor del índice de Barthel. Este hallazgo puede resultar paradójico si se compara con los resultados que muestran la asociación entre niveles altos de colesterol total y el riesgo cardiovascular en múltiples estudios ${ }^{26}$.

No obstante, de manera paradójica, en el análisis de regresión longitudinal el conjunto de pacientes con cuartiles de colesterol total (Q2 a Q4) estuvo asociado con mayor índice de Barthel, hallazgo inesperado y contrario a lo encontrado en otros estudios, que puede ser efecto del manejo del anciano frágil en la unidad geriátrica de agudos $^{13}$. Lo anterior se evidencia en los hallazgos de la figura 1, donde al comienzo todos los pacientes desarrollaron discapacidad 15 días antes del ingreso hospitalario (deterioro funcional prehospitalización), lo cual muestra baja capacidad de reserva fisiológica, resilencia, que produce una pobre capacidad de respuesta y adaptación a la enfermedad aguda, lo que refleja condición de fragilidad de los pacientes. Durante el tiempo de hospitalización hubo tendencia a la mejoría funcional (deterioro intrahospitalario), período cuando fueron intervenidos en la unidad geriátrica de agudos por el equipo especializado que trabaja de forma interdisciplinaria.

Al mes de seguimiento, una vez fueron dados de alta y resolvieron su condición aguda, hubo una tendencia de mejoría funcional en todos los pacientes, más acentuada en los grupos de menor nivel de colesterol total (Q1 y Q2). Este comportamiento se explica porque estas personas tuvieron mayor pérdida funcional al inicio de la enfermedad y al resolver su enfermedad aguda tienen mayor oportunidad de mejoría funcional, lo cual es favorecido por el manejo previo en la unidad geriátrica de agudos. Este hallazgo es importante porque los metaanálisis sólo han mostrado prevención en la DF entre la condición basal 15 días antes de la hospitalización y al alta ${ }^{13}$.

De otra parte, todavía están por determinar los mecanismos que relacionan los niveles bajos de colesterol total y la discapacidad. Una posible explicación son las múltiples enfermedades crónicas que llevan a una pérdida progresiva de la funcionalidad y condición de fragilidad, generando niveles bajos de colesterol total mediados por reducción 
progresiva en actividad física, sarcopenia y estado de inflamación crónica ${ }^{27}$.

Estudios previos realizados en ancianos no frágiles hospitalizados por enfermedad aguda, sugieren que los niveles de colesterol total $\geq 200 \mathrm{mg} / \mathrm{dl}$ se asocian con mayor probabilidad para mejorar la discapacidad en las actividades básicas de la vida diaria ${ }^{28}$. En contraste, los resultados de la investigación muestran que a partir de valores $\geq 117 \mathrm{mg} / \mathrm{dl}$ de colesterol total, existe mayor probabilidad de presentar mejores valores en el índice de Barthel durante los cuatro tiempos de observación. Este hallazgo es importante dadas las características de los participantes del estudio y es probable que la condición de fragilidad y el manejo en la unidad geriátrica de agudos lleven a que se presenten mejores resultados funcionales a pesar de tener menores niveles de colesterol total; el promedio de colesterol total en la población fue $136,2 \pm 37,9 \mathrm{mg} / \mathrm{dl}$.

En este estudio los factores asociados con mortalidad (tabla 6) fueron: sexo femenino, alta comorbilidad, demencia y estado funcional con índice de Barthel $\leq 60$. Lo anterior refleja que la mortalidad se encuentra en relación con un bajo sustrato biológico y pérdida del mecanismo de homeostasis que indican condición de fragilidad, además de deterioro mental y funcional, características que se encuentran cada vez con mayor frecuencia en los ancianos que requieren hospitalización por enfermedad aguda. Sin embargo, fue llamativo que en el estudio los niveles de colesterol total no se encontraron asociados con mortalidad pese a que diferentes investigaciones han mostrado su capacidad para predecir desenlaces adversos en salud como DF, discapacidad, deterioro cognitivo, hospitalización y mortalidad, por lo cual la capacidad predictiva del colesterol total para mortalidad en ancianos todavía permanece en controversia ${ }^{2}$.

Los hallazgos de la investigación tienen al menos tres implicaciones clínicas relevantes. La extrapolación de los resultados del estudio puede llevar a considerar que los valores elevados de colesterol total son un factor protector para la condición de salud y el estado funcional de los ancianos; por consiguiente, el beneficio del manejo con estatinas en este grupo de pacientes frágiles, con sarcopenia y discapacidad, pudiera estar cuestionado ${ }^{29}$. Así mismo, obtener la información sobre los niveles de colesterol total y el estado funcional puede mejorar la estratificación pronóstica al egreso hospitalario. Y finalmente, la valoración de la condición funcional por su capacidad de predicción pronóstica para eventos adversos en salud, se debe considerar como un signo vital en ancianos hospitalizados.

En cuanto a fortalezas, este es uno de los primeros estudios que evalúa el comportamiento de la interacción entre niveles de colesterol total y discapacidad en ancianos frágiles atendidos en una unidad geriátrica de agudos. Adicionalmente, muestra la importancia de los niveles de colesterol total sobre el estado funcional como factor clave que contribuye a la calidad de vida en ancianos hospitalizados. De igual forma, pudieron realizarse ajustes en variables conocidas como edad, comorbilidad, parámetros nutricionales como albúmina y hemoglobina y estado cognitivo, que modifican los niveles de colesterol total. Para finalizar, los intervalos de confianza estrechos de las asociaciones seleccionadas obtenidos indican un buen poder estadístico, lo cual guarda relación con el tamaño de la muestra, y, por tanto, adecuada precisión en las estimaciones e interacciones observadas, acercando los resultados al verdadero valor poblacional.

En lo concerniente a limitaciones, el estudio se realizó en ancianos frágiles atendidos en una unidad geriátrica de agudos, de modo que los resultados no pueden ser generalizados a una población diferente manejada en un modelo de atención hospitalario convencional. Aunque los análisis se ajustaron por comorbilidad y varios indicadores de estado nutricional y pobre condición de salud, es posible que el bajo nivel de colesterol total sea solo un indicador de una condición de salud pobre. En consecuencia, la asociación entre mejoría en la discapacidad para las actividades básicas de la vida diaria se deba a confusión residual por factores no medidos que no permiten detectarse mediante el análisis de la información recogida, como el efecto del manejo en la unidad geriátrica de agudos por parte de un equipo especializado, con trabajo interdisciplinario o por la presencia de enfermedad discapacitante de larga duración que llevara a una baja frecuencia de recuperación funcional. Adcionalmente, no se midieron otras fracciones del colesterol como el HDL, el cual se ha asociado con discapacidad independientemente de edad, género, comorbilidad y niveles de colesterol total ${ }^{24}$, así como tampoco se indicó si los pacientes venían recibiendo manejo con estatinas lo cual pudo haber subestimado las asociaciones ya que el manejo con estos medicamentos se indica con mayor frecuencia en ancianos que tienen mejor reserva funcional ${ }^{29}$.

\section{Conclusión}

Los resultados del estudio muestran que en ancianos frágiles hospitalizados en unidades geriátricas de agudos, el colesterol total en los cuartiles más altos estuvo asociado con mejoría en la recuperación de las actividades básicas de la vida diaria desde 15 días antes del ingreso hospitalario hasta el mes posterior al alta, comparado con el Q1 de forma independiente. Estos resultados avalan el concepto que los bajos niveles de colesterol total son un marcador de pobre estado de salud y discapacidad en ancianos hospitalizados.

\section{Responsabilidades éticas}

Protección de personas y animales. Los autores declaran que para esta investigación no se han realizado experimentos en seres humanos ni en animales.

Confidencialidad de los datos. Los autores declaran que han seguido los protocolos de su centro de trabajo sobre la publicación de datos de pacientes.

Derecho a la privacidad y consentimiento informado. Los autores declaran que en este artículo no aparecen datos de pacientes.

\section{Financiación}

Ninguna. 


\section{Conflicto de intereses}

\section{Ninguno.}

\section{Bibliografía}

1. Wiebe DA, Westgard JO. Cholesterol - a model system to relate medical needs with analytical performance. Clin Chem. 1993:39:1504-12.

2. Schatz IJ, Masaki K, Yano K, Chen R, Rodriguez BL, Curb JD. Cholesterol and all-cause mortality in elderly people from the Honolulu Heart Program: a cohort study. Lancet. 2001;358:351-5.

3. Harris TB. Cholesterol and health in old age: risk factor or risk marker? J Am Geriatr Soc. 2004;52:639-40.

4. Fried LP, Guralnik JM. Disability in older adults: evidence regarding significance, etiology, and risk. J Am Geriatr Soc. 1997; 45:92-100.

5. Bologa RM, Levine DM, Parker TS, Cheigh JS, Serur D, Stenzel Kh, et al. Interleukin- 6 predicts hypoalbuminemia, hypocholesterolemia, and mortality in hemodialysis patients. Am J Kidney Dis. 1998;32:107-14.

6. Volpato S, Zuliani G, Guralnik JM, Palmieri E, Fellin R. The inverse association between age and cholesterol level among older patients: the role of poor health status. Gerontology. 2001;47:36-45.

7. Karlamangla AS, Singer BH, Reuben DB, Seeman TE. Increases in serum non- high-density lipoprotein cholesterol may be beneficial in some high-functioning older adults: MacArthur studies of successful aging. J Am Geriatr Soc. 2004;52:487-94.

8. Vauthey C, de Freitas GR, van Melle G, Devuyst G, Bogousslavsky J. Better outcomes after stroke with higher serum cholesterol levels. Neurology. 2000;54:1944-8.

9. Fried LP, Tangen CM, Walston J, Newman AB, Hirsch C, Gottdiener J, et al. Frailty in older adults: evidence for a phenotype. J Gerontol Med Sci. 2001;56A:M146-56.

10. Hardy SE, Gill TM. Factors associated with recovery of independence among newly disabled older persons. Arch Intern Med. 2005;165:106-12.

11. Gill TM, Kurland B. The burden and patterns of disability in activities of daily living among community-living older persons. J Gerontol A Biol Sci Med Sci. 2003;58A:70-5.

12. Cornette P, Swine C, Malhomme B, Gillet JB, Meert P, D'Hoore W. Early evaluation of the risk of functional decline following hospitalization of older patients: development of a predictive tool. Eur J Public Health. 2006;16:203-8.

13. Fox MT, Persaud M, Maimets I, OB́rien K, Brooks D, Tregunno D, et al. Effectiveness of acute geriatric unit care using acute care for elders components: a systematic review and meta-analysis. J Am Geriatr Soc. 2012;60:2237-45.

14. Mahoney FI, Barthel DW. Functional evaluation: Barthel index. Md State Med J. 1965;14:61-5.
15. Folstein MF, Folstein SE, McHugh PR. Mini-mental state' . A practical method for grading the cognitive state of patients for the clinician. J Psychiatr Res. 1975;12:189-98.

16. Inouye SK, Van Dyck CH, Alessi CA, Balkin S, Siegal AP, Howitz RI. Clarifying confusion: the confusion assessment method. A new method for detection of delirium. Ann Intern Med. 1990;113:941-8.

17. Charlson ME, Pompei P, Ales KL, Mackenzie CR. A new method of classifying prognostic comorbidity in longitudinal studies: Development and validation. J Chronic Dis. 1987;40:373-83.

18. Miralles R, Sabartés O, Ferrer M, Esperanza A, Llorach I, GarcíaPalleiro P, et al. Development and validation of an instrument to predict probability of home discharge from a Geriatric Convalescence Unit in Spain. J Am Geriatr Soc. 2003;51:252-7.

19. World Health Organization Nutritional Anemia: Report of a, WHO., Scientific Group. Tech Rep Ser. 1968;405:1-40.

20. Diez-De-Los-Ríos MJ, Bermúdez RM, García SG. Estandarización de los procedimientos de medida de creatinina: estado actual. Rev Lab Clin. 2012;5:87-101.

21. Cabañero-Martínez MJ, Cabrero-García J, Richart-Martínez M, Muñoz- Mendoza CL. Revisión estructurada de las medidas de actividades de la vida diaria en personas mayores. Rev Esp Geriatr Gerontol. 2008;43:271-83.

22. Hunger $M$, Doring A, Holle R. Longitudinal beta regression models for analyzing health-related quality of life scores over time. BMC Med Res Methodol. 2012;12:144.

23. Ellis G, Whitehead MA, O’Neill D, Langhorne P, Robinson D. Comprehensive geriatric assessment for older adults admitted to hospital. Cochrane Database Syst Rev. 2011;7. CD006211.

24. Zuliani G, Romagnoni F, Bollini C, Leoci V, Soattin L, Fellin R. Low levels of high-density lipoprotein cholesterol are a marker of disability in the elderly. Gerontology. 1999;45:317-22.

25. Windler E, Ewers-Grabow U, Thiery J, Walli A, Seidel D, Greten $\mathrm{H}$. The prognostic value of hypocholesterolemia in hospitalized patients. Clin Invest. 1994;72:939-43.

26. National Cholesterol Education Program (NCEP) Expert Panel on Detection, Evaluation, and Treatment of High Blood Cholesterol in Adults (Adult Treatment Panel III). Third Report of the National Cholesterol Education Program (NCEP) Expert Panel on Detection, Evaluation, and Treatment of High Blood Cholesterol in Adults (Adult Treatment Panel III) final report. Circulation. 2002;106:3143-421.

27. Kronmal RA, Cain KC, Ye Z, Omenn GS. Total serum cholesterol levels and mortality risk as a function of age. A report based on the Framingham data. Arch Intern Med. 1993;153: 1065-73.

28. Onder G, Volpato S, Liperoti R, D’Arco C, Maraldi C, Fellin $\mathrm{R}$, et al. Total serum cholesterol and recovery from disability among hospitalized older adults. J Gerontol A Biol Sci Med Sci. 2006;61:736-42.

29. Strandberg TE, Kolehmainen L, Vuorio A. Evaluation and treatment of older patients with hypercholesterolemia: a clinical review. JAMA. 2014;312:1136-44. 